\title{
The large-angle photon veto detector system for the NA62 experiment at CERN
}

\author{
Paolo Valente $\mathrm{a}^{\mathrm{a} 1}$ \\ ${ }^{a}$ INFN Sezione di Roma, Roma, Italy
}

\begin{abstract}
The goal of the NA62 experiment is to collect about $80 K^{+} \rightarrow \pi^{+} v \bar{v}$ events with a S/B ratio of $\sim 10: 1$. The branching ratio (BR) for this decay is $\sim 10^{-10}$ and can be predicted in the Standard Model with minimal theoretical uncertainties, making it a sensitive probe for new physics. Measurement of this BR is challenging because of the background from dominant channels. To reduce background from $K^{+} \rightarrow \pi^{+} \pi^{0}$ decays (BR $=21 \%$ ) to an acceptable level, the $\pi^{0}$ must be detected with an inefficiency of less than $10^{-8}$. NA62 will make use of the existing NA48 beam-line and liquid-krypton calorimeter. A new photon veto system consisting of 12 rings placed along the vacuum volume is needed to detect large-angle photons ( 7 to $50 \mathrm{mrad}$ ) with an inefficiency below $10^{-4}$ over the energy interval from a few hundred MeV to $35 \mathrm{GeV}$. A comprehensive R\&D program was carried out in 2007-2008 to compare different detector technologies, including the re-use of lead-glass blocks from the OPAL barrel calorimeter, ultimately demonstrating the feasibility of this approach. In 2009, a complete prototype of one veto ring was constructed, complete with front-end electronics to measure energies through time-over-threshold with a dynamic range of 1000, and an in-situ calibration and monitoring system. The prototype was successfully tested with electron and muon beams at the CERN SPS in fall 2009. The status of the project and present preliminary results from the recent tests will be reviewed.
\end{abstract}

Key words: photon detector, calorimeter, veto system, Kaon, rare decay PACS: $29.40 . \mathrm{Vj}$

\section{Introduction}

The CERN NA62 experiment [1] aims at collecting about 80 $K^{+} \rightarrow \pi^{+} \nu \bar{v}$ events in two years of data-taking, keeping the background by at least a factor of 10 below the signal. For this purpose, a very intense hadron beam of $75 \mathrm{GeV} / c$ momentum, with $6 \%$ of Kaons, will be produced by $400 \mathrm{GeV} / c$ protons slowly extracted from the SPS. The $10 \mathrm{MHz}$ of kaon decays along the $120 \mathrm{~m}$ fiducial volume (in vacuum) will be detected by a series of detectors. The design of the experiment has been optimized for providing kinematical rejection of the backgrounds and particle identification.

In particular, in order to keep the $K^{+} \rightarrow \pi^{+} \pi^{0}$ background $(\mathrm{BR}=21 \%)$ at the level of $10^{-12}$, only a factor of $\sim 10^{-4}$ is achieved by kinematical cuts on the parent $K^{+}$and the daugher $\pi^{+}$tracks, so that the $\pi^{0}$ must be detected with an inefficiency below a few $10^{-8}$. The two $\gamma$ 's from the $\pi^{0}$ decay will be detected by the NA48-legacy quasi-homogenous liquid-Krypton $(\mathrm{LKr})$ calorimeter in the intermediate angular range and by a set of 12 veto stations placed all along the decay region for large angle $\gamma$ 's (large angle vetoes, LAV), as shown in the schematic layout in Fig. 1. Hermeticity will be ensured for photons inside the LKr hole by additional photon detectors after the primary beam-sweeping magnet.

In order to match the requirements, the LAV stations should detect photons above $200 \mathrm{MeV}$ and up to several tens of $\mathrm{GeV}$, in the $8.5 \leq \theta \leq 50 \mathrm{mrad}$ range, with an inefficiency below

\footnotetext{
${ }^{1}$ on behalf of the NA62 photon veto working group.
}

$10^{-4}$, providing a time-stamp with a resolution of $\sim 1 \mathrm{~ns}$ and a measurement of the deposited energy of about $10 \%$. In order to make use of beam-halo muons for calibration and efficiency studies, the detector should be sensitive to minimum ionizing particles (MIP). Finally, in order to be integrated in the decay region tank, the detectors should be capable of operation in vacuum $\left(10^{-6}\right.$ mbar in the region of the spectrometer $)$.

\section{LAV design and assembly}

For the 12 LAV stations, three possible options were tested by the collaboration: lead/scintillating fibers, annularshaped detectors, inspired by the KLOE calorimeter; rings of lead/scintillating tiles modules, read out radially by wavelength shifting fibers; rings of crystals from the OPAL experiment calorimeters, re-arranged in radial arrays. After extensive tests, all three choices were found to match the $10^{-4}$ inefficiency requirement and the OPAL-crystals solution has been selected. Details on prototypes studies can be found in Ref. [2,3].

Counters will be mounted in each ring with a radial orientation. As the inner radius of curvature of the blocks is not matched to the radius of the rings, several layers of blocks will be used. As an example, the first five rings, with an inner radius of $53 \mathrm{~cm}$, will be composed of five layers, 32 blocks each. Each layer is shifted by a fifth of the angle between two blocks in order to have complete hermeticity: at least three blocks $\left(20 X_{0}\right)$ are crossed by each inpinging particle.

The crystals are fixed to aluminum support structures in groups of four, forming azimuth segments which are then fixed 


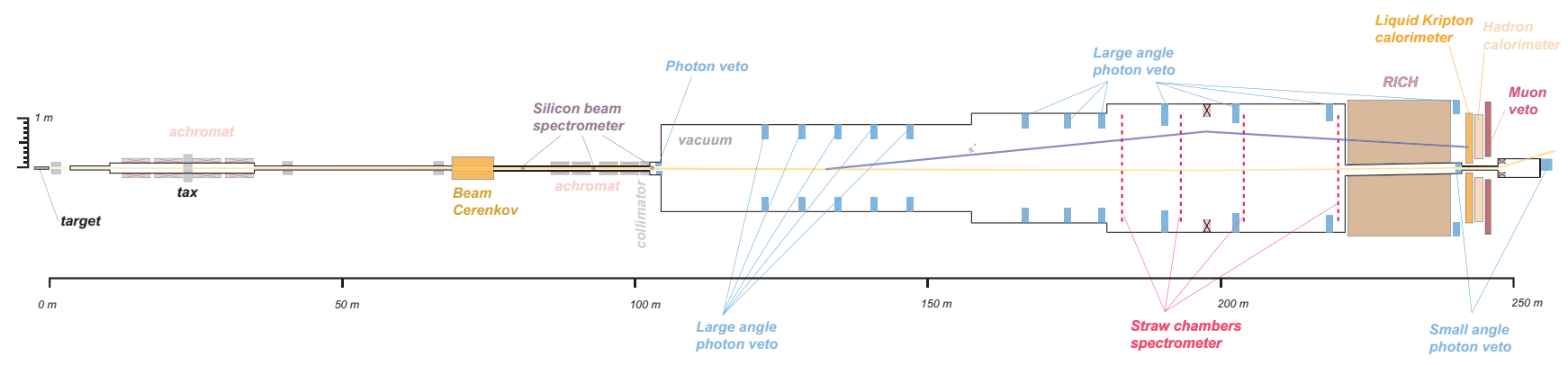

Figure 1: Schematic layout of the NA62 experiment. The 12 LAV stations all along the decay vacuum tube are shown.

to the inner surface of a stainless-steel cylinder to form the five layers of crystals, protruding towards the center of the cylinder. The steel cylinders will be inserted into the main tank of NA62 to form the $120 \mathrm{~m}$ long vacuum decay volume.

Every crystal has its own photomultiplier, Hamamatsu $\mathrm{R} 2238$, which has to operate in vacuum, and an optical connector for the connection of a LED light source, for calibration and monitoring purposes. The analog signals from the photomultipliers are connected to vacuum feed-throughs housed on flanges (ISO K200) on the cylindrical vessel through coaxial cables.

In Fig. 2 all the steps of the assembly of one veto ring are shown: each lead-glass crystal is wrapped using $0.2 \mathrm{~mm}$ thick DuPont Tyvek pre-cut sheets, in order to increase light collection; then crystals are mounted in groups of four in the support brackets; after having routed $\mathrm{HV}$ and signal cabling to the PMT's, the support brackets are placed and fixed to the inner surface of the ring vessel (with the cylinder held in vertical position) and the cables are routed to the portholes of flanges. Once the mounting of crystals is completed, the vessel is rotated by $90^{\circ}$ and vacuum $\&$ electrical tests are performed.

\section{LAV electronics}

This wide photon energy range, together with the necessity of detecting muon signals, requires operating the R2238 PMTs at a gain of order $10^{6}$. The average photoelectron yield of the crystals is 0.3 p.e./MeV. This translates in a $4.5 \mathrm{pC}$ charge for a MIP, corresponding to a signal amplitude of $20 \mathrm{mV} / 50 \Omega$. On the upper part of the range, signals from $20 \mathrm{GeV}$ showers can reach an amplitude of $10 \mathrm{~V} / \Omega$. Such a signal range would be outside the possibilities of commonly used ADC chips, whose dynamic range is at most 50. On the other hand, the range of a typical amplifier-shaper-discriminator chain is of order 10.

In selecting the readout scheme, the cost and data throughput must also be considered. Moreover, since we should build a veto system, a simple and robust solution is desirable. Since the requirement of signals as high as a few volts rules out most commonly used ASICs produced for HEP applications, our idea is to use commercial amplifier and comparator circuits to produce a digital output signal with time duration equal to that of the analog input (time-over-threshold), exploiting the advantage of having high-amplitude signals and a relatively slow rise time.
Using this technique, the resulting dynamical range of the input signal is enlarged due to the typical non-linear relationship between the time-over-threshold width and the input signal amplitude. A picture of oscilloscope traces for two PMT analog signals and corresponding LVDS time-over-threshold signals is shown in Fig. 3.

A clamp stage has been also added to the design, in order to protect the electronics from very large signals while keeping the time development of the signal untouched. The digital signal from the discriminator is then transmitted to the TDC using the LVDS differential system. The TDC and acquisition board will be the same as in most of the other experimental subsystems (the TELL1 board originally developed for the $\mathrm{LHCb}$ experiment).

\section{Installation in NA62 and beam test}

Once the first ring of LAV was assembled in Frascati, the complete veto station was transported to CERN and installed in the existing iron vacuum vessel (the "blue tube") by removing a $6 \mathrm{~m}$ long section and fixing the LAV steel cylinder by means of one fixed and one sliding flange (vacuum-tight). We have performed extensive vacuum tests and long-term operation of PMT's in vacuum. For the purpose, we have arranged a prototype HV control system for powering all the 160 crystals in the ring module.

We have also prepared a prototype readout system, in order to fully equip one half of the ring ( 80 crystals), so that we were able to perform extensive tests with muon and electron beams during SPS running in summer 2009.

The prototype readout system was mainly intended for the validation of the front-end electronics design and built for our detector. In particular, each signal coming from the LAV PMT's was fed to prototype boards (with VME mechanics and power supply) of the time-over-threshold discriminators. The boards include an amplification stage with dual output: one copy of the signal is fed to the discriminator, which produces LVDS digital signals with time-over-threshold duration, while a second copy is available for routing to a different readout.

For the charge measurement, we have used commercial 12bit charge ADC, $400 \mathrm{pC}$ range (CAEN V792). In order to provide a gate for $\mathrm{ADC}$ integration, a trigger signal was provided by the fast-OR of the 16 digital signals in the first, i.e. upstream, row (out of five) of lead-glasses. 

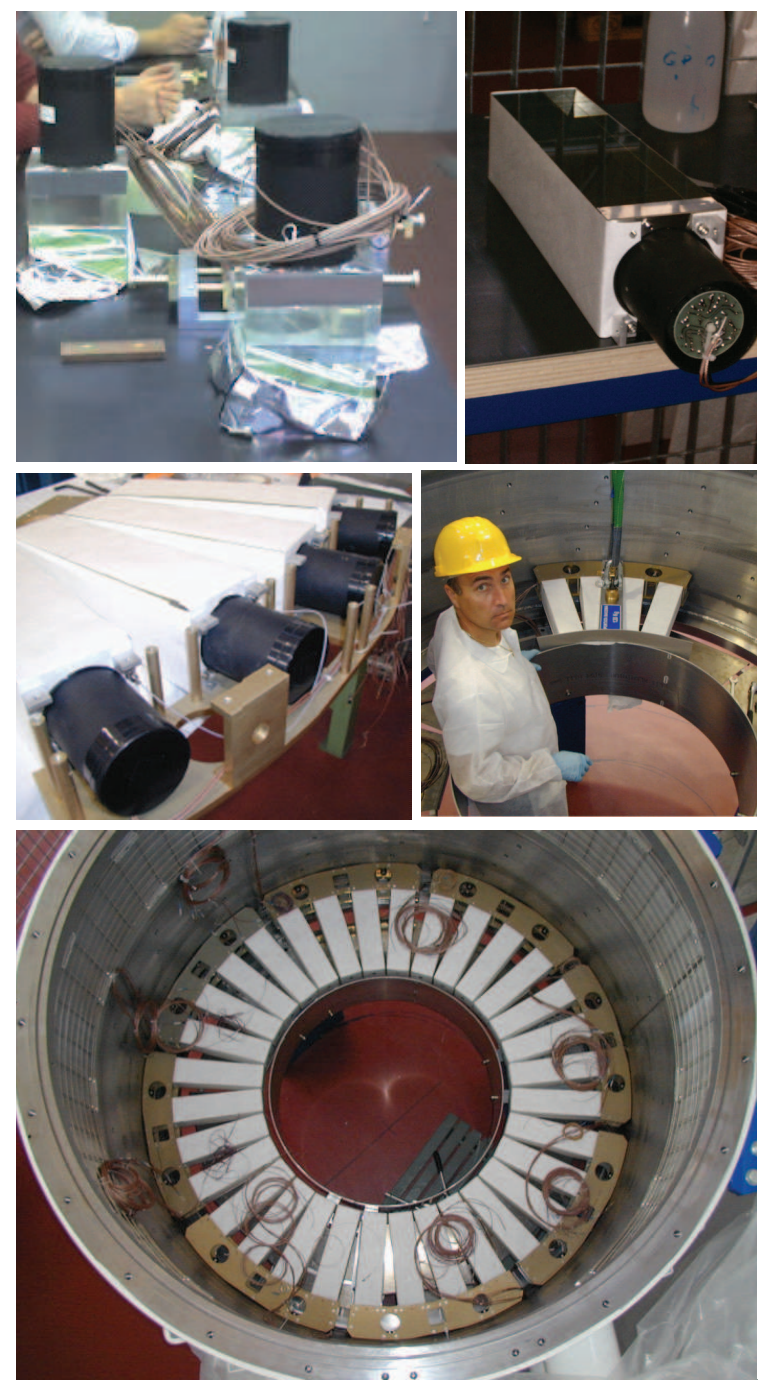

Figure 2: LAV station assembly phases (from top-left): cabling of PMT, wrapping of crystals, mounting of four-crystals brackets, fixing of brackets inside the steel cylinder, routing of cables to vacuum flange ports.

A stand-alone DAQ system was also prepared, capable of gating the acquisition with SPS status signals and sustaining a trigger rate of about $1 \mathrm{kHz}$. Raw data were written first to local disk, and then automatically transferred to network-attached storage. An automatic reconstruction program was also developed in order to produce TDC and QDC histograms for offline analysis, as well as an online graphical display of rates on each crystal.

We have collected large samples of data both with a diffuse halo of muons, thus illuminating all the counters in the ring (obtained by closing the TAX collimators upstream in the K12 beam-line), and with electron beams of energy 2, 4 and $6 \mathrm{GeV}$. Due to the dimensions of the electron beam (few $\mathrm{cm}^{2}$ ), we have performed several scans by means of trim magnets in order to hit the equipped half ring in different positions.

Muon data were used first for checking the gain equalization of the PMT's, already performed before the installation with cosmic rays test-stands in Naples and Frascati. As shown in

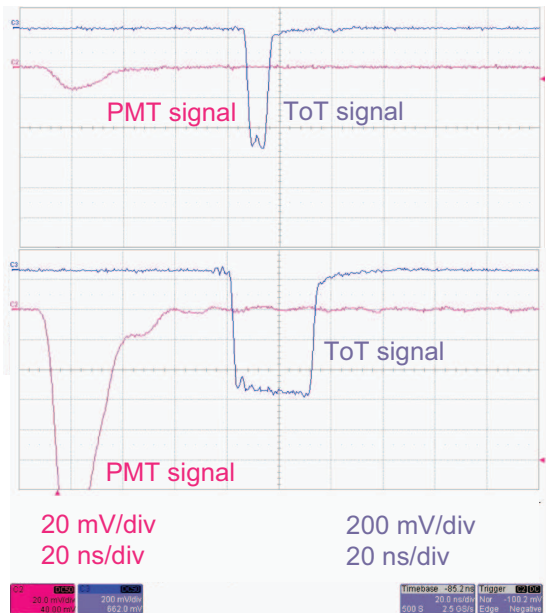

Figure 3: Analog signal from PMT and LVDS signal from time-over-threshold discriminator for two muon signals, ranging from $\sim 20$ (top) and $\sim 600 \mathrm{mV} / \Omega$ (bottom).

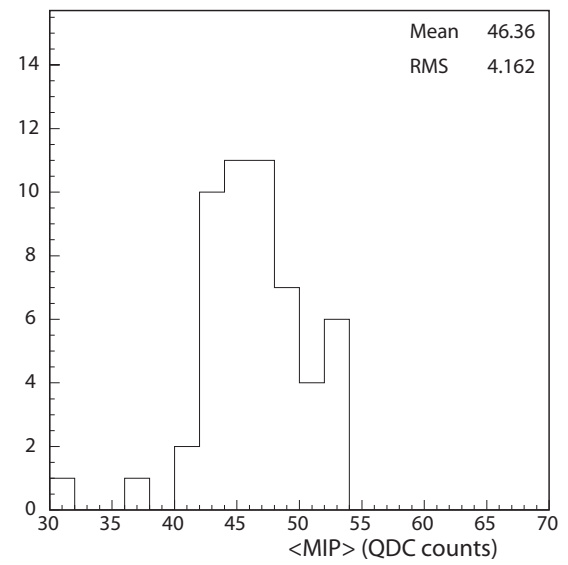

Figure 4: Average values of MIP for all equipped lead-glass crystals for a muon run in NA62 (summer 2009). The HV settings were adjusted on the basis of cosmic-rays data. The mean of $45 \mathrm{ADC}$ counts corresponds to $4 \mathrm{pC}$, well matching what is expected for a gain of $\sim 10^{6}$, the RMS is at $10 \%$ level.

Fig. 4, the average value of MIP for all the crystals is $\sim 4 \mathrm{pC}$, as expected for a gain of about $10^{6}$, with a RMS at the level of $10 \%$ using nominal HV settings, as shown in Fig. 4.

Using muon data we also performed a calibration of the timeover-threshold discriminator threshold: we have collected data at $7,10,15$ and $25 \mathrm{mV} / 50 \Omega$ for the input signal, corresponding to a range from 0.3 to $\sim 1$ MIP. For each crystal, we measure the fraction of events with at least one valid TDC hit as a function of the charge measured by the ADC, as shown in Fig. 5, together with the energy scale deduced from the gain calibration: it is clear that we can safely set a threshold at a fraction of the MIP value.

Using electron data we were able to check the performance of the time-over-threshold technique by comparing the time width of the LVDS output of the discriminator (measured by the TDC) with the charge measured by the ADC (Fig. 6). The correlation is excellent, except for a fraction of events (in the circle in the figure) for which the time-over-threshold measurement 


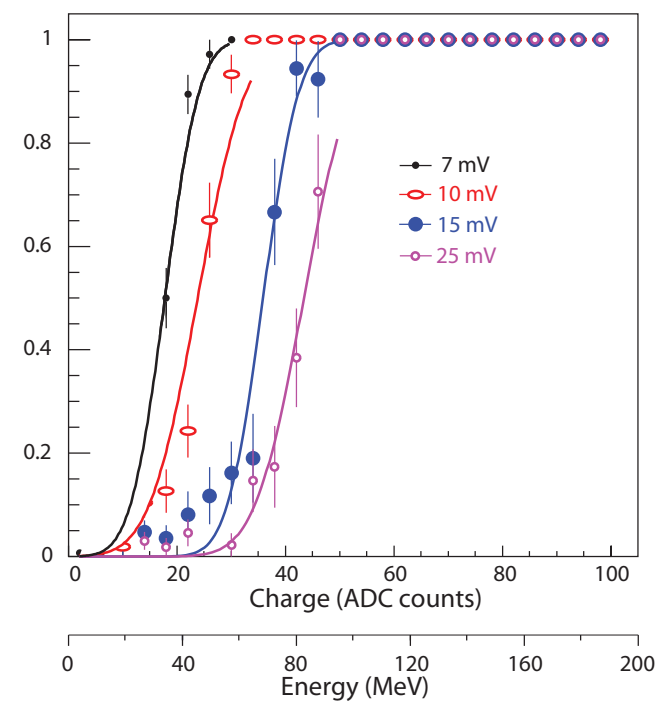

Figure 5: TDC efficiency as a function of signal amplitude (recorded by the charge ADC) for four different values of the threshold of time-over-threshold discriminator. Taking into account the MIP energy deposit $(\sim 80 \mathrm{MeV})$ and the MIP/ADC calibration factor, the corresponding energy scale has been calculated.

is lengthened: this is due to the ringing of the analog signal, caused by a small impedance mismatch in the PMT's (now being fixed in the HV divider circuit), which in turn determines one or more refirings of the discriminator. When the leading and trailing edges of digital signals are too close, the TDC is not able to resolve different time hits $(<7.5 \mathrm{~ns})$, so that the time-width is lengthened (by $\sim 10 \mathrm{~ns}$ ).

\section{References}

[1] G. Anelli et al., CERN/SPSC 2005-013 (2005).

[2] F. Ambrosino et al., IEEE NSS 2007, arXiv:0711.3398 (2007).

[3] F. Perfetto et al., Nucl. Phys. B (Proc. Suppl.) 197224 (2009).

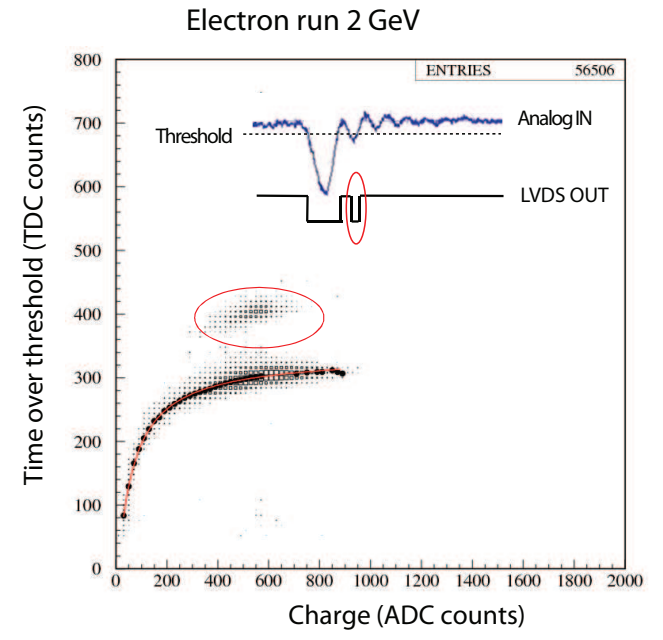

Figure 6: Time width $(1 \mathrm{TDC}$ count $=0.1 \mathrm{~ns})$ from the time-over-threshold output of the discriminator as a function of the signal charge measured by the ADC for electron $(6 \mathrm{GeV})$ events. The ringing of the analog signal, due to a small impedance mismatch in the PMT's (now being fixed in the HV divider circuit), is visible as lengthening of the time-over-threshold width for a fraction of events (in the circle) when the TDC is not capable of resolving too close leading and trailing digital signal edges. 